\title{
Laparoscopic splenectomy in pediatric age: long-term follow-up
}

\author{
Ufuk Ates, M.D. ${ }^{a}$, Nil Y. Tastekin, M.D. ${ }^{a}$, Gulnur Gollu, M.D., Assist Prof. ${ }^{a}$, \\ Ergun Ergun, M.D. ${ }^{a}$ and Aydin Yagmurlu, Prof. ${ }^{a}$
}

\begin{abstract}
Introduction: In the last century, with the advancement of the diagnostic procedures, hematologic disorders in pediatric age group have been increased dramatically. In parallel with this increase, splenectomy procedures have also been popularized with different techniques and surgical outcomes. Laparoscopic splenectomy (LS) in the pediatric age group is generally accepted as a technically demanding procedure, which needs experience. The purpose of this study is to present thelong-term follow-up results of a case series of children who underwent LS for a variety of hematologic disorders, evaluate possible complications and outcomes.

Population and methods: All patients who were admitted to the clinic and who were scheduled for LS from 2005 to 2016 were considered for this study. The study parameters were grouped in four categories including socio-demographic data, preoperative evaluation, clinical follow-up and complications.

Results: There were 24 male $(48.9 \%)$ and $25(51.1 \%)$ female patients. The median age and body weight for the study group was 12 years and $35 \mathrm{~kg}$. Most common indications for LS were thalassemia $(13 ; 26.5 \%)$ and hereditary spherocytosis $(12 ; 24.4 \%)$. As a technical standpoint, 2 patients (4\%) underwent singleport LS surgery. The mean time for LS surgeries was found as 80 minutes. The total intraoperative complication rate was $4 \%(2 / 57)$. The mean time for hospital stay was 5 days. Mean follow-up period was 6.4 years (range: 6 months-16 years). There was no long-term complication. Bilirubin levels and need for blood transfusion significantly decreased in the long term follow-up period $(\mathrm{p}<0.05)$.

Conclusions: LS is a powerful tool in the hands of an experienced surgeon. It's a safe and effective procedure in children with hematologic disorders resulting in shorter length of stay and lower complication rates.

Key words: child, follow-up studies, laparoscopy, splenectomy.
\end{abstract}

http:/ / dx.doi.org/10.5546/aap.2017.eng.e385

Assist. Prof. Gulnur Gollu: drggollu@yahoo.com

Funding:

None.

Conflict of interest:

None.

Received: 1-16-2017

Accepted: 7-16-2017
To cite: Ates U, Tastekin NY, Gollu G, et al. Laparoscopic splenectomy in pediatric age: long-term follow-up. Arch Argent Pediatr 2017;115(6):e385-e390.

\section{INTRODUCTION}

In the last century, with the advancement of the diagnostic procedures, hematologic disorders in pediatric age group have been increased dramatically. In parallel with this increase, splenectomy procedures have also been popularized with different techniques and surgical outcomes..$^{1-6}$ Laparoscopic splenectomy (LS) in the pediatric age group is generally accepted as a technically demanding procedure, which needs experience. $^{7-11}$

Although widely used, the studies analyzing the outcomes for LS surgeries are very inconclusive in the literature. ${ }^{12}$ The purpose of this study is to present the long-term followup results of a case series of children who underwent LS for a variety of hematologic disorders, evaluate possible complications and outcomes.

\section{POPULATION AND METHODS}

\section{Study population}

All patients who were admitted to the clinic and who were scheduled for LS from 2005 to 2016 were considered for this study. After protocol review, the data collection was granted by the chairman of the pediatric surgery department and all guidelines for confidentiality were followed throughout the retrospective data collection procedure.

As an inclusion criterion, only LS (primary LS combined with or without cholecystectomy) were included in the study group. Exclusion criteria for the study were: patients who had multiple surgeries, patients who scheduled for any open surgeries regarding gastrointestinal system and partial splenectomy cases whether open or laparoscopically performed. 
During enrollment, a total of 57 cases were enrolled for the study. Hematologic diagnosis of children including the splenomegaly were established in the pediatric hematology department. After the establishment of the diagnosis of splenomegaly, all patients underwent ultrasonographic examination in order to rule out the cases that had accessory spleens and determine the size of the spleen. If ultrasound examination was inconclusive, then selective spleen scintigraphy was applied for the definitive diagnosis. Preoperatively, all patients received a pneumococcal polyvalent vaccine at least two weeks before the surgery. Patients were transfused preoperatively if their $\mathrm{Hb}$ levels were $<10 \mathrm{~g} / \mathrm{dL}$. Eight open splenectomy cases, were excluded from the final analysis. As a result, 49 cases were found to be eligible. All operations were performed by two surgeons experienced for the laparoscopic procedures. Brief description of the operative technique is previously described in the literature. ${ }^{7}$

\section{Study parameters}

All patients' data collection was retrospectively performed by two investigators blinded to the clinical outcomes. All patients' medical charts were reviewed according to the study protocol and all study parameters were recorded in computer software for further analysis.

The study parameters for the cases were grouped mainly in four categories including socio-demographic information (age, sex, body weight), indication for LS, preoperative evaluation (including the laboratory results and radiographic outcomes), clinical follow-up and any complications. For preoperative evaluation, all diagnostic procedures and medications were determined including any radiographically work-ups. Laboratory results before and after surgery including infection parameters (white blood cell count, c-reactive protein, erythrocyte sedimentation rate), complete blood cell count (haemoglobin, haematocrit, white blood cell and platelet count), abdominal ultrasonography and if any selective spleen scintigraphy results were checked from the medical records. After surgery, the clinical follow-up visits were evaluated and duration of hospital stays, duration of surgeries and re-surgeries if any needed were recorded. The intraoperative and postoperative complications including any recurrence, infection, wound drainage, abscess or any mortality were also noted for further analysis.

\section{Long-term follow-up and statistical analysis}

Patients were called for control one month after surgery and after the first month, the followup was scheduled at 6 months and annually, thereafter. The long-term follow-up was carried out by the analysis of the medical charts of these patients who had regular visits to our department. A special medical record was created at the final follow-up for the long-term analysis including the mortality, any re-operations due to any adhesions or bridles, laboratory values including preoperative and postoperatvie blood hemoglobin levels, platelet cell count and bilirubin levels including total and direct bilirubin, accidental intraperitoneal splenic self-implantation or any infection including pneumonia or meningitis. Finally, the need for blood transfusions pre and postoperatively in the follow-up period was also recorded.

Five different statistical analysis were performed for the long-term follow up analysis. Patients' blood hemoglobin levels, platelet cell counts, total and direct bilirubin levels and the need for blood transfusions were compared between preoperative and postoperative controls including the final follow-up visit. For the statistical comparison of all parameters except the need for blood transfusion, Friedman's two-way analysis of variance (ANOVA) test was used. For the statistical analysis of the need for blood transfusion Wilcoxon signed rank test was used. The results of statistical analysis were expressed as number of observations $(\mathrm{n})$, mean \pm standard deviation $( \pm)$, median and minimum-maximum values [M (min-max)]. SPSS Ver.17.0 (SPSS Inc., Chicago IL, USA) and MedCalc 13.1.0.0 (MedCalc Software bvba, Ostend, Belgium) statistical package programs were used for the analysis of data set. $p<0.05$ was considered statistically significant.

\section{RESULTS}

There were 24 male $(48.9 \%)$ and 25 female $(51.1 \%)$ patients. The median age and body weight for the study group was 12 years (range: $3-18$ years) and $35 \mathrm{~kg}$ (range: $12-72 \mathrm{~kg}$ ),

TABLE 1. Demographic features of the study group

\begin{tabular}{lccc}
\hline \multirow{2}{*}{ Demographic features } & \multicolumn{2}{c}{ Gender (\%) } & \multirow{2}{*}{ Total } \\
\cline { 2 - 3 } & Male: & Female: & \\
& $\mathbf{3 0 ~ ( 5 2 . 6 )}$ & $\mathbf{2 7}(\mathbf{4 7 . 4 )}$ & \\
\hline Median age (years) & 14 & 10 & 12 \\
Median body weight (kg) & 35 & 33 & 35 \\
Mean spleen size (cm) & 14.2 & 13.5 & 13.9 \\
\hline
\end{tabular}


respectively. There were a total of three cases $(6.1 \%)$ between 10 to $15 \mathrm{~kg}$ of body weight in the study group (Table 1). Most common indications for LS surgeries were thalassemia $(13,26.5 \%)$ and hereditary spherocytosis $(12$, $24.4 \%$ ) (Table 2). All cases were undergoing a meticulous hematological consultation before the LS including laboratory studies. There was no increase in any of the infection parameters before surgery for all cases. Additionally, all patients underwent abdominal ultrasonographic examination in order to rule out the accessory spleens. For five cases whose ultrasonographic examination revealed no accessory spleen, selective spleen scintigraphy was performed $(n=5)$. The mean size of the spleens was $13.9 \mathrm{~cm}$ $(5,5-23 \mathrm{~cm})$.

LS in combination with cholecystectomy were performed in 16 patients $(32.6 \%)$. The indication for cholecystectomy was cholelithiasis for all patients. As a technical standpoint, two patients (4\%) underwent single-port LS. Hand-assisted splenectomy was performed because the size of the spleen in one of the patients was too large to fit into the specimen bag.

TABLE 2. Indications for the laparoscopic splenectomy

\begin{tabular}{lcc}
\hline Indications & Frequency & Percentage \\
\hline Thalassemia & 13 & 26.5 \\
Hereditary spherocytosis & 12 & 24.5 \\
Autoimmune hemolytic anemia & 9 & 18.4 \\
Sickle cell disease & 6 & 12.2 \\
Idiopathic thrombocytopenic purpura & 3 & 6.2 \\
Others* & 6 & 12.2 \\
\hline
\end{tabular}

${ }^{*}$ Hematolohical malignencies, pulmonary hypertension, severe combined immune deficiency, spleen mass.
The mean time for LS surgeries was 80 minutes (40-300 minutes). The total intraoperative complication rate was $4 \%(2 / 49)$. One patient had prolonged surgery (approximately 300 minutes) due to massive hemorrhage and technical issues related to single port LS. There was no additional complication for this patient in the postoperative follow-up period. The other patient had bronchopulmonary spasm intraoperatively. After proper management in the intensive care unit, this patient was discharged from the hospital with no additional morbidities.

The mean time for hospital stay was five days (4- 11 days). There was only one patient who was hospitalized more than one week due to latex allergy leading to intraoperative bronchopulmonary spasm, which was aforementioned previously. In the early postoperative period, there was only one recurrent abscess formation (total postoperative complication rate $2 \%$ ) which was treated by drainage and debridement with laparotomy.

\section{Long-term outcomes}

Mean follow-up period was 6.4 years (range: 6 months - 16 years). There were no re-operations due to any adhesions or bridles. All cases had normal blood cell counts at the final followup which were monitored by the hematology department. There was also no postoperative mortality in the follow-up period. There was no accidental intraperitoneal splenic selfimplantation or any recorded infection including pneumonia or meningitis through the follow-up period.

The mean values and statistical comparison results of the study parameters including the blood hemoglobin levels, platelet cell counts,

TABLE 3. The outcomes for long-term analysis of the study parameters before and after surgery. Mean values

\begin{tabular}{lcccccc}
\hline Study Parameters & Preoperative & \multicolumn{2}{c}{ Postoperative } & \multicolumn{2}{c}{$p$ value } \\
\cline { 2 - 6 } & & 1st month & 6th month & 1st year & Final control & \\
\hline Hemoglobin (g/dL) & 10,56 & 11,72 & 11,64 & 11,42 & 11,78 & 0,453 \\
Platelet (x10^6/L) & 294894 & 424139 & 453900 & 455200 & 438500 & 0,068 \\
Total bilirubin (mg/dL) & 2,60 & 1,15 & 1,56 & 1,78 & 1,62 & 0,002 \\
Direct bilirubin (mg/dL) & 1,00 & 0,48 & 0,94 & 1,13 & 0,86 & 0,004 \\
\hline
\end{tabular}

TABLE 4. The need blood transfusions annually in the long-term follow up period before and after surgery.

\begin{tabular}{lcccc}
\hline The need for blood transfusion & Preoperative & \multicolumn{2}{c}{ Postoperative } & \multirow{2}{*}{$p$ value } \\
\cline { 3 - 4 } & & 1st year & Final control & \\
\hline Annual mean values & 8.2 & 1.3 & 1.7 & 0.037 \\
\hline
\end{tabular}

*The mean number of refferal for blood transfusion 
bilirubin levels and the need for blood transfusions before and after surgery were summarized in Table 3 and 4. Although there was no significant difference for the blood hemoglobin levels and platelet cell counts before and after surgery ( $p=0.321$ and 0.578 , respectively), all bilirubin levels (direct and total) were found significantly lower in the long term follow-up period ( $p<0.05$ ). The need for blood transfusions were also significantly decreased for all patients after surgery $(p<0.05)$ and stayed in that manner through the follow-up period.

\section{DISCUSSION}

LS is a powerful tool in the hands of an experienced surgeon. It is a safe and effective procedure in children with hematologic disorders resulting in shorter length of stay, and lower complication rates. With the advent of minimally invasive techniques, laparoscopic splenectomy has become a standard procedure for elective removal of the spleen for most indications. It is commonly accepted in the literature that LS is a technically difficult procedure and is associated with a steep learning curve, with operative times initially longer than those associated with open splenectomy. Nevertheless, technical advancements such as hand-assisted laparoscopic splenectomy have facilitated increased acceptance of laparoscopy as an approach that is superior to open splenectomy, even in patients with severe hematologic diseases associated with massive splenomegaly. ${ }^{12-14}$ In this study, one patient underwent hand assisted splenectomy and this technique can be used instead of open splenectomy when needed.

Currently, three major laparoscopic techniques have been used for the splenectomy surgeries. These include multi-port, single-port and handassisted LS surgeries. The European Association for Endoscopic Surgery(EAES) identifies multiport LS as the gold standard of treatment for surgical diseases involving normal to moderately enlarged spleens. ${ }^{1}$ A meta-analysis by Winslow and Brunt ${ }^{15}$ found that despite its longer duration, multi-port LS was associated with a shorter postoperative stay in hospital, lower complication rates and fewer pulmonary, wound and infectious complications. Other systematic reviews ${ }^{16,17}$ also show the superiority of LS over conventional open splenectomy. So, it is very commonly accepted in the literature that LS had significantly lower mortality and complication rates. For this reason, in the current study, LS was preferred as the primary treatment choice for the patients with splenomegaly due to hematological disorders. The authors believe that, laparoscopic approach should be thought first in hand for all cases scheduled for splenectomy.

There is still an ongoing debate in the literature about the real indications and possible drawbacks of single-port LS. ${ }^{18}$ The major drawbacks about the single-port surgery discussed in the literature are the problems with triangulation, clashing of instruments and lack of space. So, there should be a standardization of the single-port splenectomy technique as well as better patient selection criteria. Single-port umbilical LS has been described in children and has been shown to have excellent cosmetic advantages compared with conventional LS. ${ }^{19}$ Nevertheless, there has yet to be large-scale prospective randomized studies directly comparing standard multiport LS with single-port splenectomy, differences between instruments and differences between surgical techniques in terms of patient outcomes..$^{20}$ In the current study, single-port splenectomy was applied to two children. These two surgeries took longer than the conventional LS but there were no additional complications with better cosmetic outcomes. We believe that, surgeon experience is the mainstay for the choice of the technique and clear guidelines must be published for the singleport splenectomy surgeries.

In the literature, although there are dozens of studies analyzing the outcomes for LS, there is indeed still a discussion about the gold standard surgical technique for splenectomy in children. In one of the early studies by Esposito et al., 54 children who underwent LS were analyzed and as a conclusion it was stated that the operating time of LS is still too long compared with open surgery, and the extraction phase still not simple enough. ${ }^{21}$ The authors also stated that perfect control of hemostasis is fundamental because severe complications can arise from even a slight bleeding episode. With the evolution and refinement of LS techniques, this procedure becomes effective even in patients with massive spleens and previous abdominal surgery. In a study by Park et al., a multi-center research was conducted analyzing the effectiveness of LS. ${ }^{22}$ In this study, a total of 203 splenectomy cases were evaluated and in conclusion it was stated that LS is both safe and feasible in patients of all ages and for a wide range of splenic pathologies. Our experience with LS is also consistent with the literature. We believe that laparoscopic exposure 
should be the first choice for all hematologic cases scheduled for splenectomy with low morbidity and high success rates.

Shorter length of hospital stay and the shorter operative time are two distinguished features of the LS, commonly emphasized in the literature. In a study by Targorona et al., the mean operative time and length of hospital stay for LS was reported as 153 minutes and four days, respectively for a total of 113 cases. ${ }^{23}$ In another study by Rescorla et al., the mean hospital stay was reported as 1.4 days and the means operative time was found as 115 minutes. ${ }^{18}$ In a recent study by Deng et al., 57 pediatric cases with LS were analyzed and it was reported that the mean length for hospital stay was five days and the mean operative time was 120 minutes. ${ }^{13}$ Although in this study for length of hospital stay were consistent with the literature with an average of five days, this is not the case for the length of operative time. Most of the studies and the ones summarized above reported over 100 minutes of average operative time for LS. ${ }^{12,24,25}$ On contrary, in our study, the average operative time was reported as 80 minutes. This difference mainly depends on the experience of the surgical team to the minimally invasive procedures.

One of the potential advantages of LS is the low complication rates due to less aggressive nature of the approach and the great visual details obtained. In the literature, large series analyzing the rates of LS for hematologic disorders repeatedly report a significant decrease in complication rates compared with the conventional open approach even in the longterm follow-up..$^{22,26,27}$ Nevertheless, majority of these studies are retrospective case series and randomized trials are lacking. Most authors report lower operative blood loss with LS. Postoperative splenectomy complications, such as pneumonia and atelectasis, were less frequent with LS than OS. ${ }^{12}$ Postoperative patient recovery times were also shorter with LS. Finally, patients who had LS returned to regular activities more quickly and had better cosmetic results than patients who had OS. The benefits of LS are also valid for the long-term follow-up. Corcione et al., reported no complication of LS in a 5 year follow-up period with significantly lower rates of transfusion. ${ }^{28}$ In another study by Lesher et al., with a mean follow-up of 5.6 years, LS was found a safe and effective procedure with significantly lower hospital re-admission rates after surgery. ${ }^{14}$ The study of Patkowski et al. also analyzed the long-term results of LS with a maximum 8 years of follow-up. There was only one complication 16 months after surgery in their series and the authors concluded that LS should be accepted as the gold standard treatment method for spleen disorders. ${ }^{3}$ The long-term results of the current study have comparable results with the literature. The current study has a mean follow-up period of 6.4 years with a maximum 16 years of follow-up. This is one of the longest follow-up periods in the literature. In our study, we had only two intra-operative and one early post-operative complication (recurrent abscess formation). Massive intra-operative hemorrhage and bronchopulmonary spasm are two common intraoperative complications that must be kept in mind during the LS. There was no longterm complication and a significant decrease of bilirubin levels and the need for blood transfusion was detected in the post-operative period.

There are some limitations of the current study. First of all, this is a retrospective case series with no randomized open surgical group to compare. Second, on final evaluation the effect of spleen size was not analyzed. There is also no comparison of single versus multiport surgical techniques including the ones that were combined with cholecystectomy. Finally, our study population is not large enough to have precise conclusions about the outcomes of LS with limited outcome measures. Hence, this study must be considered as a pilot study for the other large series with more comprehensive data analysis.

\section{CONCLUSION:}

LS is a powerful tool in the hands of an experienced surgeon. It is a safe and effective procedure in children with hematologic disorders resulting in shorter length of stay, and lower complication rates. Future prospective studies are needed in order to have a precise conclusion about the real indications and long-term outcomes of LS.

\section{REFERENCES:}

1. Habermalz B, Sauerland S, Decker G, et al. Laparoscopic splenectomy: the clinical practice guidelines of the European Association for Endoscopic Surgery (EAES). Surg Endosc 2008;22(4):821-48.

2. Murawski M, Patkowski D, Korlacki W, et al. Laparoscopic splenectomy in children--a multicenter experience. JPediatr Surg 2008;43(5):951-4.

3. Patkowski D, Chrzan R, Wróbel G, et al. Laparoscopic splenectomy in children: experience in a single institution. J Laparoendosc Adv Surg Tech A 2007;17(2):230-4. 
4. Bala M, Edden $Y$, Mintz $Y$, et al. Blunt splenic trauma: predictors for successful non-operative management. Isr Med Assoc J 2007;9(12):857-61.

5. Berman RS, Feig BW, Hunt KK, et al. Platelet kinetics and decreased transfusion requirements after splenectomy for hematologic malignancy. Ann Surg 2004;240(5):852-7.

6. Fagundes ED, Ferreira AR, Roquete ML, Penna FJ, et al. Clinical and laboratory predictors of esophageal varices in children and adolescents with portal hypertension syndrome. J Pediatr Gastroenterol Nutr 2008;46(2):178-83.

7. Delaitre B, Maignien B. Splenectomy by the laparoscopic approach. Report of a case. Presse Med 1991;20(44):2263.

8. Pattenden CJ, Mann CD, Metcalfe MS, et al. Laparoscopic splenectomy: a personal series of 140 consecutive cases. Ann R Coll Surg Engl 2010;92(5):398-402.

9. Owera A, Hamade AM, Bani Hani OI, et al. Laparoscopic versus open splenectomy for massive splenomegaly: a comparative study. J Laparoendosc Adv Surg Tech A 2006;16(3):241-6.

10. Qureshi FG, Ergun O, Sandulache VC, et al. Laparoscopic splenectomy in children. JSLS 2005;9(4):389-92.

11. Sammour T, Poole G, Bartlett A, et al. Laparoscopic splenectomy at Middlemore Hospital, New Zealand: a safe procedure with heterogeneous indications. $N$ Z Med J 2006;119(1230):U1879.

12. Gamme G, Birch DW, Karmali S. Minimally invasive splenectomy: an update and review. Can J Surg 2013;56(4): 280-5.

13. Deng XG, Maharjan A, Tang J, et al. A modified laparoscopic splenectomy for massive splenomegaly in children with hematological disorder: a single institute retrospective clinical research. Pediatr Surg Int 2012;28(12):1201-9.

14. Lesher AP, Kalpatthi R, Glenn JB, et al. Outcome of splenectomy in children younger than 4 years with sickle cell disease. J Pediatr Surg 2009;44(6):1134-8.

15. Winslow ER, Brunt LM. Perioperative outcomes of laparoscopic versus open splenectomy: a meta-analysis with an emphasis on complications. Surgery 2003;134(4):647-53.
16. Kojouri K, Vesely SK, Terrell DR, et al. Splenectomy for adult patients with idiopathic thrombocytopenic purpura: a systematic review to assess long-term platelet count responses, prediction of response, and surgical complications. Blood 2004;104(9):2623-34.

17. Mikhael J, Northridge K, Lindquist K, et al. Short-term and long-term failure of laparoscopic splenectomy in adult immune thrombocytopenic purpura patients: a systematic review. Am J Hematol 2009;84(11):743-8.

18. Rescorla FJ, West KW, Engum SA, et al. Laparoscopic splenic procedures in children: experience in 231 children. Ann Surg 2007;246(4):683-7.

19. Bruzoni M, Dutta S. Single-site umbilical laparoscopic splenectomy. Semin Pediatr Surg 2011;20(4):212-8.

20. Targarona EM, Lima MB, Balague $C$, et al. Single-port splenectomy: Current update and controversies. J Minim Access Surg 2011;7(1):61-4.

21. EspositoC,SchaarschmidtK, Settimi A, etal.Experience with laparoscopic splenectomy. J Pediatr Surg 2001;36(2):309-11.

22. Park A, Marcaccio M, Sternbach M, et al. Laparoscopic vs open splenectomy. Arch Surg 1999;134(11):1263-9.

23. Targarona EM, Espert JJ, Bombuy E, et al. Complications of laparoscopic splenectomy. Arch Surg 2000;135(10):1137-40.

24. RiceHE, CrarySE, LangerJC, etal.Comparative effectiveness of different types of splenectomy for children with congenital hemolytic anemias. J Pediatr 2012;160(4): 684-9.

25. Rosen M, Brody F, Walsh RM, et al. Outcome of laparoscopic splenectomy based on hematologic indication. Surg Endosc 2002;16(2):272-9.

26. Friedman RL, Hiatt JR, Korman JL, et al. Laparoscopic or open splenectomy for hematologic disease: which approach is superior? J Am Coll Surg 1997;185(1):49-54.

27. Targarona EM, Espert JJ, Cerdán G, et al. Effect of spleen size on splenectomy outcome. A comparison of open and laparoscopic surgery. Surg Endosc 1999;13(6):559-62.

28. Corcione F, Pirozzi F, Aragiusto G, et al. Laparoscopic splenectomy: experience of a single center in a series of 300 cases. Surg Endosc 2012;26(10):2870-6. 